\title{
Bioinspired Polyethersulfone Membrane Design via Blending with Functional Polyurethane
}

\author{
Mei Han, ${ }^{1,2}$ Qiang Liu, ${ }^{1,2}$ Baihai Su, ${ }^{1,2}$ Shudong Sun, ${ }^{1}$ and Changsheng Zhao ${ }^{1}$ \\ ${ }^{1}$ College of Polymer Science and Engineering, State Key Laboratory of Polymer Materials Engineering, Sichuan University, \\ Chengdu 610065, China \\ ${ }^{2}$ Department of Nephrology, West China Hospital, Sichuan University, Chengdu 610041, China
}

Correspondence should be addressed to Baihai Su; subaihai@scu.edu.cn and Changsheng Zhao; zhaochsh70@163.com

Received 1 October 2016; Revised 28 November 2016; Accepted 27 December 2016; Published 13 February 2017

Academic Editor: Yin Chen

Copyright (C) 2017 Mei Han et al. This is an open access article distributed under the Creative Commons Attribution License, which permits unrestricted use, distribution, and reproduction in any medium, provided the original work is properly cited.

\begin{abstract}
Polyurethanes (PUs) are currently considered to be biocompatible materials but limited by a low resistance to thrombus. We therefore design a heparin-like PU (HLPU) to modify polyethersulfone (PES) membranes approaching integrated antifouling and antithrombotic properties by bioinspiration of heparin structure. Poly(vinyl pyrrolidone)-HLPU (PVP-HLPU) was synthesized via reversible addition-fragmentation chain transfer polymerization of VP using PU as a macroinitiator and then sulfonated by concentrated $\mathrm{H}_{2} \mathrm{SO}_{4}$. FTIR and NMR results demonstrated the successful synthesis of PVP-HLPU. By incorporation of PVP-HLPU, the cross-sectional structure of PES composite membranes altered from finger-like structure to sponge-like structure resulting in tunable permeability. The increased hydrophilicity verified by water contact angles benefited both the permeability and antifouling property. As a consequence, the composite membranes showed good blood compatibility, including decreased protein adsorption, suppressed platelet adhesion, lowered thrombin-antithrombin III generation, reduced complement activation, and prolonged clotting times. Interestingly, the PVP-capped HLPU showed better blood compatibility compared to polyethyleneglycol-capped and citric acid-capped HLPUs. The results demonstrated the enhanced antifouling and antithrombotic properties of PES hemodialysis membranes by the introduction of functional HLPUs. Also, the proposed method may forward the fabrication of hemocompatible membranes via bioinspired surface design.
\end{abstract}

\section{Introduction}

Polyurethanes (PUs) are currently facing growing applications in biomedical fields such as bone regeneration [1], nearinfrared imaging [2], shape memory [3], anti-inflammatory platform [4], cancer gene therapy [5], controlled drug delivery [6], tissue engineering [7], and nervous system repair [8], due to the main advantage of their flexible chemical structure. Simple alterations of stoichiometry and/or monomers during the synthesis of PUs can produce various materials ranging from elastic thermoplastic polymers to rigid thermoset ones [9]. Besides these, a facile modification of pendent groups of PU can result in a broad spectrum of properties ranging from removing toxin bilirubin to antibacterial properties [10]. However, a limitation of low resistance to thrombi is still associated with these biomaterials that restricted their uses as blood-contacting materials.
Heparin is a highly acidic, polyanionic, and dispersive linear polysaccharide [11]. The bearing of highly negative charged groups (sulfate and carboxylate groups) and the acceptably hydrophilic structure facilitate its anticoagulant property, leading to a wide use in clinical practice. Therefore, numerous approaches have been developed to prepare heparin-immobilized PUs for potential biomedical applications with improved biocompatibility and anticoagulant activity [12], enhanced antiadhesive and antibacterial properties [13], inhibited protein and platelet adhesion [14], enhanced viability of transplanted hepatocytes and inducing angiogenesis [15], and so on. In an earlier study, bloodcontacting PU films were prepared by alternatively immersing PU films in human serum albumin and heparin solutions [16]. However, the physically coated heparin onto PUs is not stable with the ionic interaction between human serum albumin (HSA) and heparin. Thereafter, researches turn 
the direction towards covalently immobilization of heparin onto PUs. Tan et al. covalently linked heparin onto PU surfaces by the reaction between the amino group from pregrafted polyethylenimine and the carboxyl group from heparin, which significantly improved the hydrophilicity and hemocompatibility of the materials [12]. However, fabricating a heparin-like structure in PU polymer chains is rarely targeted. Herein, we aim to design novel heparin-like PU (HLPU) for the modification of polyethersulfone (PES) membrane by a simple blending method, which may be used for the fabrication of hollow dialysis membranes.

PES has become one of widely used polymers, due to its good oxidative, thermal and hydrolytic stabilities, and good mechanical and film-forming properties [17, 18]. However, the anticoagulant and antifouling properties of PES are not ideal so far. Therefore, our recent study focused on the functionalization of PES dialysis membranes by HLPUs. The heparin-like polyurethanes contained $-\mathrm{SO}_{3} \mathrm{H},-\mathrm{COOH}$, and $-\mathrm{OH}$ groups, which were fabricated to modify the anticoagulate property of PES membranes by a blending method [19]. In order to endow PES membrane with both anticoagulant and antifouling properties, herein, poly(vinyl pyrrolidone) (PVP) is employed as an end-capped groups in HLPU. The resultant PVP-HLPU was then blended with PES. The water contact angle, antifouling property, and hemocompatibility for the membranes were performed. In addition, the effect of the end-capped groups on the properties of modified PES membranes was studied.

\section{Experimental}

2.1. Materials. Polyethersulfone (PES, Ultrason E6020P) was purchased from BASF chemical company (Germany). NVinyl-pyrrolidone (VP; 99\%) was purchased from Alfa Aesar. Diphenyl-methane-diisocyanate (MDI; 98\%) and dimethylolpropionic acid (DMPA; 98\%) were obtained from Aladdin (China). MDI, DMAP, VP, and DMAc were distilled under reduced pressure prior to use. $\mathrm{N}, \mathrm{N}$-dimethylacetamide (DMAc; 98\%) was obtained from Chengdu Kelong Company (China). Bovine serum albumin (BSA) and bovine serum fibrinogen (FBG) were purchased from Sigma (USA). All the other chemicals were obtained from Chengdu Kelong Inc., China.

2.2. Synthesis and Characterization of PVP-HLPU. PU was firstly synthesized. Typically, $0.060 \mathrm{~mol}$ MDI was dissolved in $200 \mathrm{~mL}$ DMAc with stirring under $\mathrm{N}_{2}$, followed by adding $0.062 \mathrm{~mol}$ DMPA. After completely dissolving the monomers, the reaction was performed in airtight equipment at $70^{\circ} \mathrm{C}$ for $4 \mathrm{~h}$ under $\mathrm{N}_{2}$. The reaction pathway is presented in Scheme 1 . The crude product was purified by methanol and hot deionized (DI) water, respectively, to remove the residues. The obtained PU was dried completely at $40^{\circ} \mathrm{C}$ in a vacuum oven over 3 days.

Then, PU RAFT agent was synthesized. Typically, $1.74 \mathrm{~g}$ chain transfer agent (CTA), $1.18 \mathrm{~g}$ EDC, and $0.83 \mathrm{~g}$ HOBT were dissolved in $200 \mathrm{~mL}$ DMAc with continuous stirring at nitrogen atmosphere. After the activation for $1 \mathrm{~h}, 18 \mathrm{~g}$ PU was added to above-mentioned solution and kept the reaction at
TABLE 1: The compositions of modified PES membranes.

\begin{tabular}{lccc}
\hline Sample code & PES (wt.\%) & PVP-HLPU (wt.\%) & DMAc (wt.\%) \\
\hline M-0 & 18 & 0 & 72 \\
M-2 & 18 & 2 & 70 \\
M-4 & 18 & 4 & 68 \\
M-6 & 18 & 6 & 66 \\
M-8 & 18 & 8 & 64 \\
\hline
\end{tabular}

room temperature for $24 \mathrm{~h}$. The product was purified with methanol and hot DI water, respectively, to guarantee a full removal of the impurities. The obtained product (macroRAFT agent) was dried completely at $30^{\circ} \mathrm{C}$ in a vacuum oven until reaching constant weight.

Polymerization of VP into the polymer chain of PU was carried out in a sealed tube. Briefly, $2.49 \mathrm{~g}$ of VP, $1 \mathrm{~g}$ of the macro-RAFT agent, and $0.02 \mathrm{~g}$ of AIBN were added to a Schlenk flask followed by the addition of DMAc. After three cycles of freeze-pump-thaw, the reaction mixture was allowed to warm to $80^{\circ} \mathrm{C}$ under a nitrogen atmosphere, and the polymerization was carried out for $10.5 \mathrm{~h}$. After dialysis against DI water for couple of days, the product was freezingdried resulting in white powders and termed as PVP-PU.

The obtained PVP-PU was sulfonated by concentrated $\mathrm{H}_{2} \mathrm{SO}_{4}$ with a mass ratio of $1: 10$. After stirring at room temperature in the $\mathrm{H}_{2} \mathrm{SO}_{4}$ for $10 \mathrm{~h}$, the PVP-HLPU was purified with DI water for couple of times to remove the residue $\mathrm{H}_{2} \mathrm{SO}_{4}$. The HLPU powder was dried at $30^{\circ} \mathrm{C}$ for $72 \mathrm{~h}$. A Fourier transform infrared spectroscope (FTIR) and a BRUKER spectrometer were used to characterize PVP-HLPU polymer.

2.3. Preparation and Characterization of Membranes. The membranes were prepared by a liquid-liquid phase-inversion technique as described in our earlier studies [20]. The modified membranes with different mass percentages of the PVP-HLPU are shown in Table 1.

A scanning electron microscope (FE-SEM, JSM-7500F, JEOL, Japan) was used to characterize the cross section morphology of the membranes.

The hydrophilicity of the membranes was characterized by a contact angle goniometer (OCA20, Dataphysics, Germany) equipped with video capture. At least five measurements were averaged to reach a reliable value.

2.4. Antifouling Property of Membranes. The characterization of the antifouling property of membranes were provided in S1 (see Supporting Material available online at https://doi.org/10.1155/2017/2158124).

2.5. Blood Compatibility of Membranes. The protein adsorption behavior of PES membranes was carried out according to our previous study [10]. The protein adsorption, platelet adhesion, clotting times (activated partial thromboplastin times (APTTs) and thrombin times (TTs)), platelet activation (Human Platelet Factor 4 (PF4)), coagulation activation (thrombin-antithrombin III complex (TAT)), and complement activation ( $\mathrm{C} 3 \mathrm{a}$ and $\mathrm{C} 5 \mathrm{a}$ ) of PES membranes were 


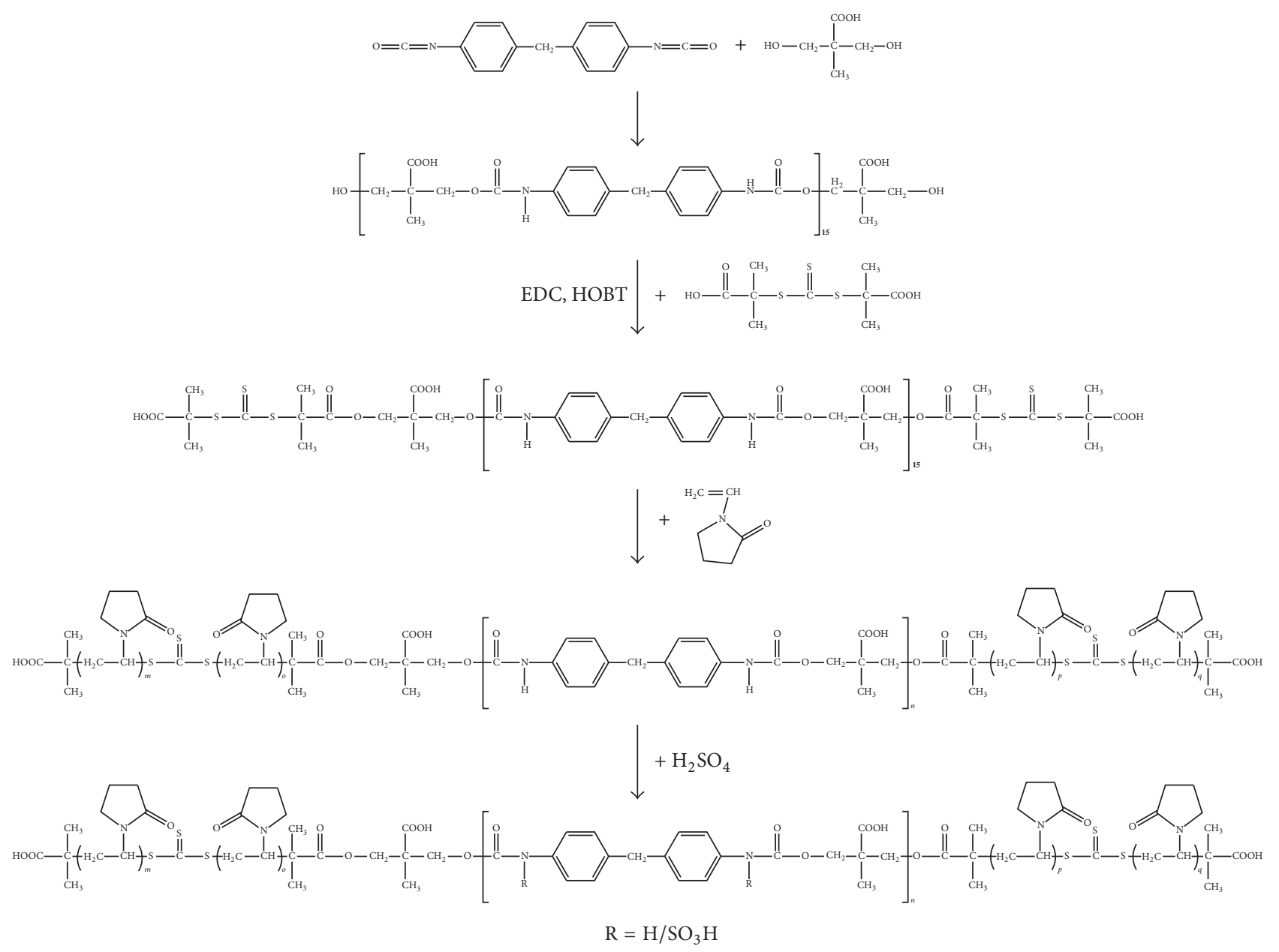

SCHEMe 1: Synthesis of PVP-HLPU.

carried out, and the details were provided in S2 (see Supplementary Material).

\section{Results and Discussion}

3.1. Synthesis and Characterization of HLPU. The FTIR spectra for the PVP-PU and PVP-HLPU are shown in Figure 1. It was observed that the peaks at $3310 \mathrm{~cm}^{-1}$ and $3391 \mathrm{~cm}^{-1}$ were the characteristic stretching vibration peaks of the $\mathrm{N}-$ $\mathrm{H}$ of the isocyanate [19] in the PVP-PU and PVP-HLPU, respectively. After the sulfonation, the peak intensity at $3310 \mathrm{~cm}^{-1}$ decreased, which indicated the successful sulfonation of PVP-PU. The peaks at $2914 \mathrm{~cm}^{-1}$ and $2908 \mathrm{~cm}^{-1}$ were attributed to the $-\mathrm{CH}_{3}$ of the CTA in the PVP-PU and PVP-HLPU, respectively, while the peaks at 1732 and $1736 \mathrm{~cm}^{-1}$ were attributed to the $-\mathrm{C}=\mathrm{O}$ in the carboxyl group of the isocyanate in both the PVP-PU and PVP-HLPU. The characteristic stretching vibration peak of cyclanone in VP was observed at $1662 \mathrm{~cm}^{-1}$. Besides, the characteristic peak of $\mathrm{S}=\mathrm{O}$ of the sulfonic group in PVP-HLPU should be between 1600 and $1750 \mathrm{~cm}^{-1}$, which was covered by the characteristic peak of $\mathrm{C}=\mathrm{O}$.

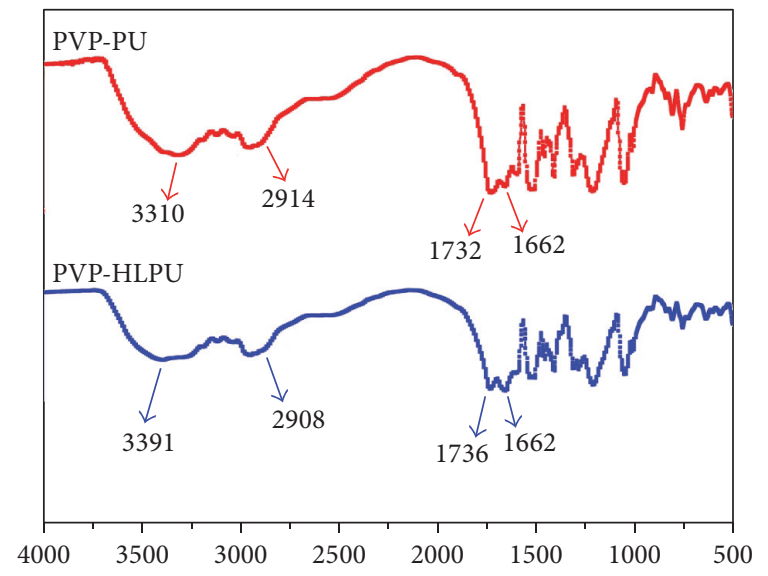

FIgURE 1: FTIR spectra of PVP-PU and PVP-HLPU.

The NMR spectra for the PVP-PU and PVP-HLPU are presented in Figure 2. The synthesized PVP-PU was verified by the signals at a $(\delta=9.59 \mathrm{ppm}, \mathrm{N}-\mathrm{H}), \mathrm{b}$ and $\mathrm{c}(\delta=$ 7-7.5 ppm, $\mathrm{Ar}-\mathrm{H}), \mathrm{d}\left(\delta=4.22 \mathrm{ppm},-\mathrm{CH}_{2}-\right.$ in DMPA $)$, e 

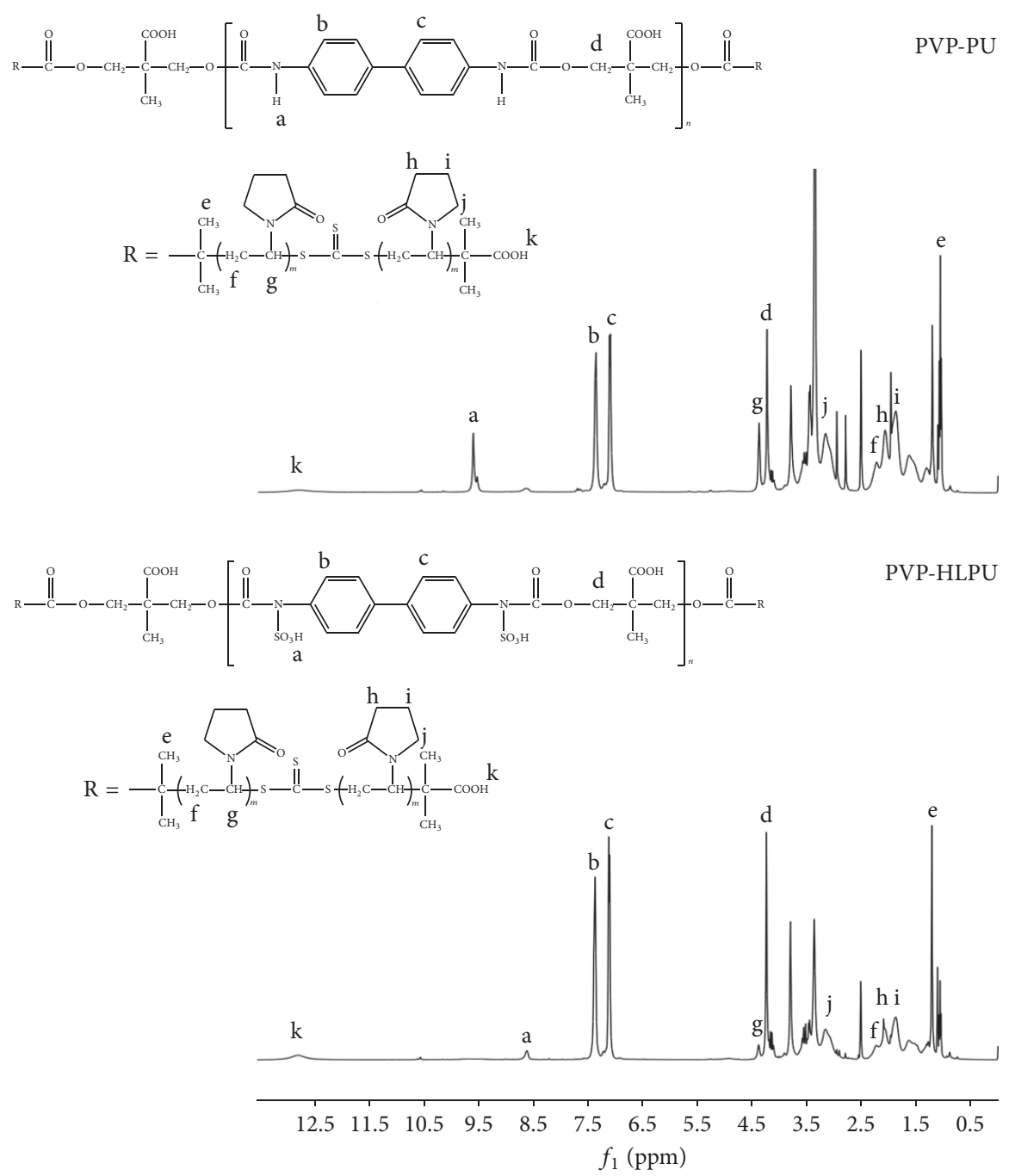

Figure 2: The ${ }^{1} \mathrm{H}$ NMR of PVP-PU and PVP-HLPU.

( $\delta=1.21 \mathrm{ppm},-\mathrm{CH}_{3}$ of the CTA), $\mathrm{f}\left(-\mathrm{CH}_{2}-\right.$ on the PVP backbone), $g$ (C-H on the PVP backbone), $h, i$, and $j$ (the $-\mathrm{CH}_{2}$ - of the cyclanone in VP), and $\mathrm{k}(-\mathrm{COOH}$ of the PVP$\mathrm{PU})$. Compared to the NMR spectrum for the PVP-PU, the signal at $\delta=9.59 \mathrm{ppm}(\mathrm{N}-\mathrm{H})$ of the NMR spectrum for the PVP-HLPU almost disappeared, which was replaced with an intensified signal at $\delta=8.53 \mathrm{ppm}$ due to the sulfonic group in PVP-HLPU).

3.2. Morphologies and Hydrophilicity of PES Membranes. Figure 3 shows the SEM images for the cross section of the membranes. It was observed that the cross section structure of the membranes showed two dense layers and an interlayer. Interestingly, the pores of the modified membranes changed gradually from finger-like structure to sponge-like structure with the addition of the PVP-HLPU, when compared to the membrane M-0. Especially when the PVP-HLPU content reached $8 \mathrm{wt} . \%$ (M-8), the finger-like pores almost disappeared and the cross-sectional structure nearly changed to sponge-like structure. Furthermore, the sponge-like pore size increased gradually with the PVP-HLPU content changing from $2 \mathrm{wt} . \%$ to $8 \mathrm{wt} . \%$; the reason might be that the hydrophilic PVP-HLPU migrated to the membrane surfaces and the pore surfaces during the phase separation process of the membrane preparation [17].

3.3. Antifouling Property of Membranes. To investigate the antifouling property of the membranes, BSA ultrafiltration was carried out, and the data are shown in Figure 4. The PBS fluxes of all the modified membranes were higher than that of the pristine PES membrane. For the modified membranes, the PBS flux increased with increasing the HLPU amount, which might be resulted from the changed membrane structure and the increased surface hydrophilicity (as shown in Figure 3 and Table 2). It was reported that the viscosity of the casting solution would be changed after blending the amphiphilic PU, which had great effect on the phase separation during membrane formation [21].

Meanwhile, a slight reduction in the PBS solution was observed with time as shown in Figure 4. The fluxes of 

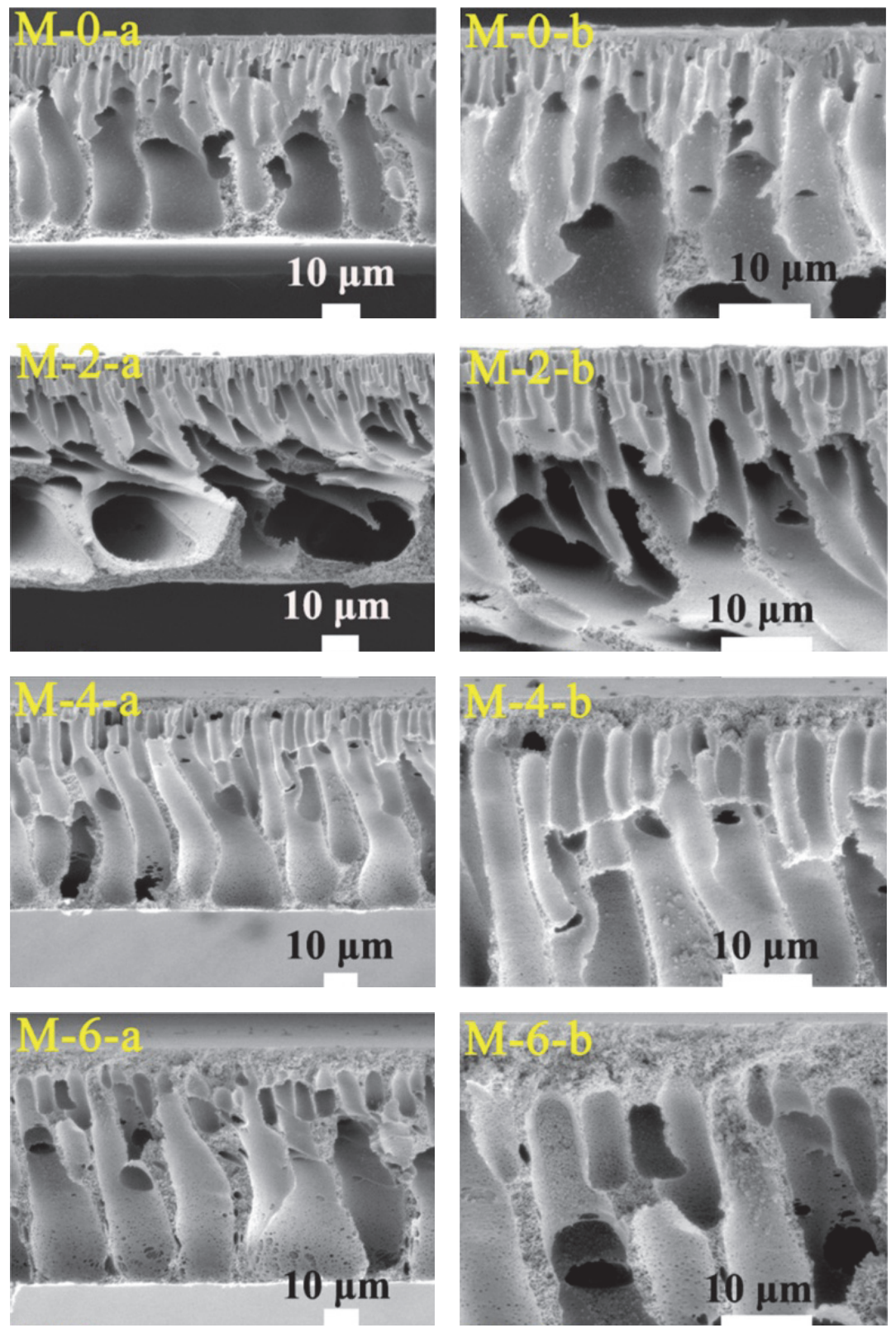

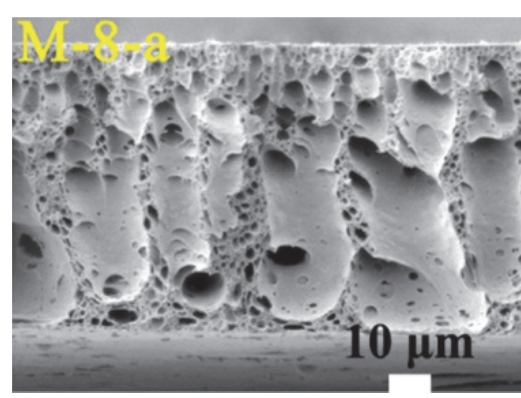

(a)

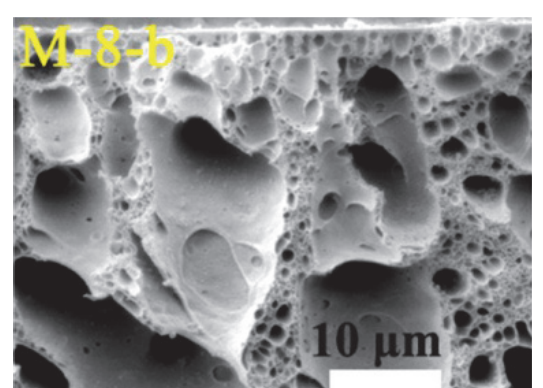

(b)

FIgURE 3: SEM images of the cross sections of different PES membranes. Magnification: (a) 1000x; and (b) 2500x. 
TABLE 2: Water contact angle and permeability of PES membranes.

\begin{tabular}{lcccc}
\hline Sample code & Copolymer $($ wt. \%) & Water contact angle $\left(^{\circ}\right)$ & Water flux $\left(\mathrm{mL} / \mathrm{h} \times \mathrm{m}^{2} \times \mathrm{mmHg}\right)$ & Flux recovery ratio $(\%)$ \\
\hline M-0 & 0.0 & $76.8 \pm 2.2$ & 30.6 & 40.8 \\
M-2 & 2.0 & $65.8 \pm 1.9$ & 90.0 & 81.7 \\
M-4 & 4.0 & $58.2 \pm 1.5$ & 113.6 & 84.7 \\
M-6 & 6.0 & $51.3 \pm 1.8$ & 133.5 & 88.2 \\
M-8 & 8.0 & $46.7 \pm 2.5$ & 155.1 & 92.0 \\
\hline
\end{tabular}

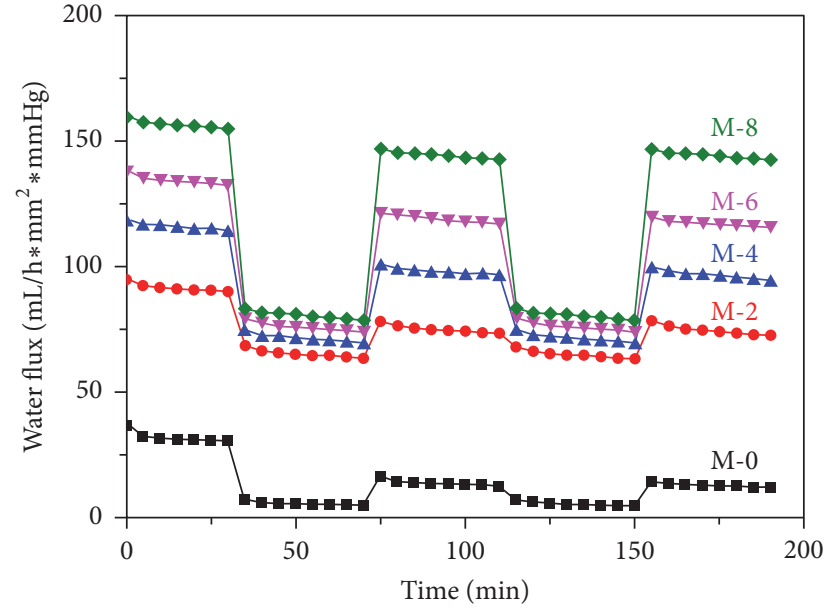

Figure 4: Time-dependent fluxes of the PES and modified membranes. PBS solution: 0-30 min, 80-110 min, and 160-190 min; BSA solution: $40-70 \mathrm{~min}$ and $120-150 \mathrm{~min}$.

the membranes decreased dramatically when the filtration solution changed from PBS to BSA solution. This might be due to BSA molecules deposition/adsorption on the membrane surfaces and/or in the membrane pore surfaces [22].

The decline in flux under constant pressure was caused by two reasons: concentration polarization and membrane fouling [23]. As a result, several cycles of filtration and flux recovery ratios were tested to determine the main reason for the flux decline of the membranes. The composite membranes were washed with PBS solution for $10 \mathrm{~min}$ after $60 \mathrm{~min}$ of protein ultrafiltration. Afterward, the PBS solution fluxes were carried out once again. It could be observed in Figure 4 that the fluxes for all the modified membranes recovered in a larger extent compared to the pristine PES membrane after rinsing in DI water for $30 \mathrm{~min}$. It indicated that the fluxes of the modified membranes were much easier to be recovered and thus showed better antifouling property.

In addition, flux recovery ratios $\left(F_{\mathrm{RR}}\right)$ were used to judge the antifouling property [24]. The better antifouling property of the membrane is reflected by higher $F_{\mathrm{RR}}$ values. As shown in Table 2, all $F_{\mathrm{RR}}$ values for the modified membranes were over $80 \%$, which are higher than that $(40.8 \%)$ of the pristine PES one. In addition, $F_{R R}$ increased gradually with increasing blended amounts of HLPU. It indicated that the composite membranes showed good antifouling property after the addition of HLPU.

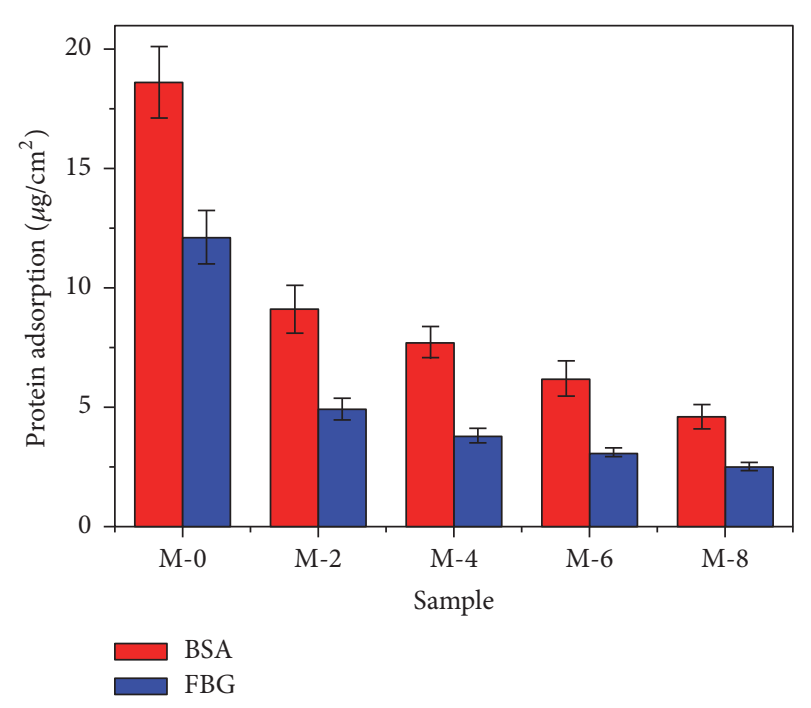

FIGURE 5: The protein adsorbed amount for the pure PES membrane and the composite membranes.

\subsection{Blood Compatibility of Membranes}

3.4.1. Protein Adsorption. Protein adsorption on biomaterial surfaces is always considered as the first step of many undesired bioreactions and bioresponses [25]. Protein adsorption is affected by numerous factors, such as hydrophilicity/hydrophobicity, surface charge, surface topology, interactions between the adsorbed molecules, the composition of the protein solution, and the surface chemistry [26]. The modified membranes after blending with hydrophilic additives exhibited decreased protein adsorption and enhanced biocompatibility [22]. Otherwise, plasma protein adsorbed on material surface had great influence on platelet adhesion and activation [27]. Thus, protein adsorption was firstly tested to investigate the blood compatibility of the membranes.

The adsorption of BSA and FBG of the membranes was studied in vitro, and the results are presented in Figure 5. As expected, all the modified membranes exhibited decreased protein adsorption amounts compared to the pristine PES membrane $(\mathrm{M}-0)$. The BSA adsorption and FBG adsorption for the pristine PES membrane were $18.5 \mu \mathrm{g} / \mathrm{cm}^{2}$ and $11.8 \mu \mathrm{g} / \mathrm{cm}^{2}$, respectively. As for the modified membranes, the protein adsorption amounts decreased with increasing the blended HLPU content. In particular, when the PVPHLPU content was $8 \mathrm{wt} . \%$, the BSA and FBG adsorption of the membrane (M-8) decreased dramatically to $4.9 \mu \mathrm{g} / \mathrm{cm}^{2}$ and $2.4 \mu \mathrm{g} / \mathrm{cm}^{2}$, respectively. It might be caused by the 

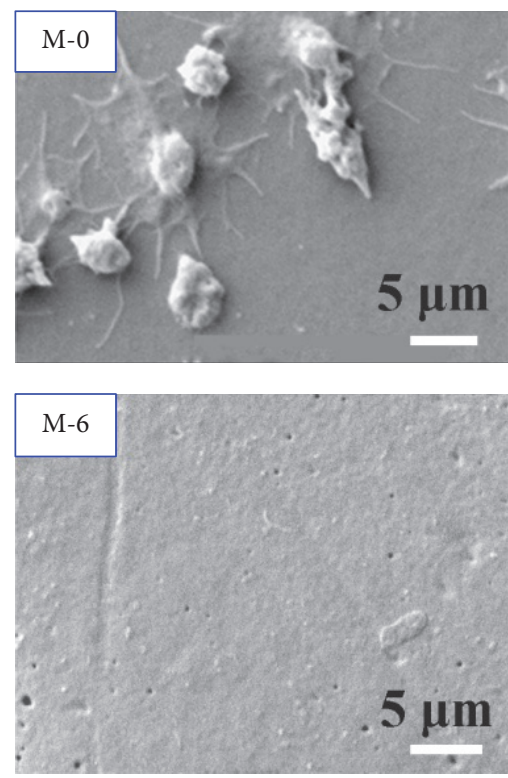
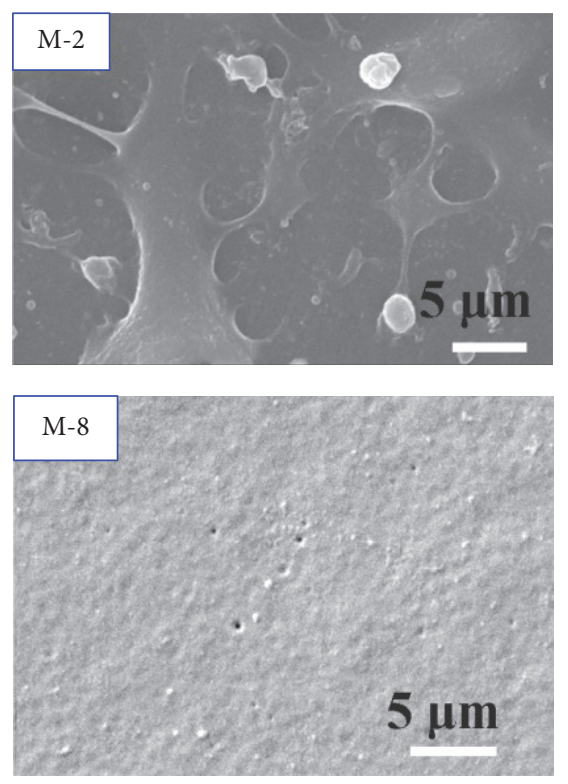
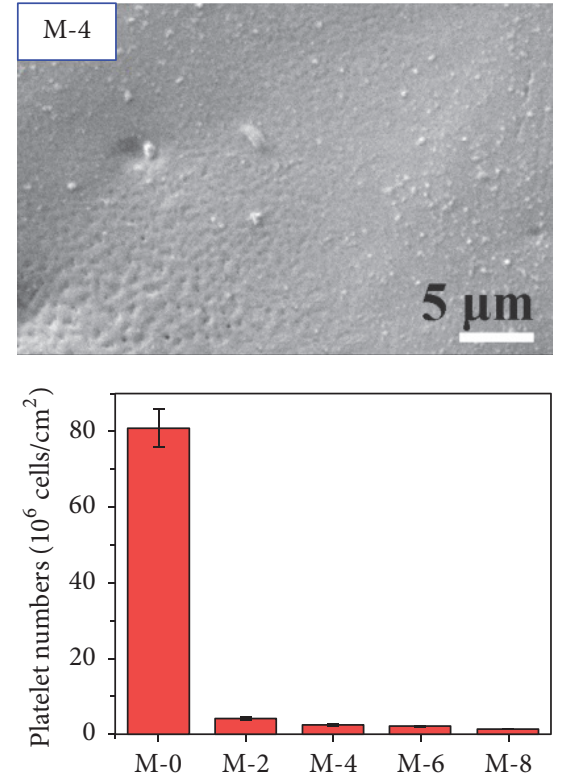

FIGURE 6: SEM micrographs of platelets adhered on the membrane surface.

increased hydrophilicity and electrostatic repulsion of the negative charge of the modified membrane surface. The enhanced protein antifouling properties improved the blood compatibility of the modified membranes.

3.4.2. Platelet Adhesion. Platelet adhesion on the surface of biomaterials is also critical to evaluate hemocompatibility. Once a foreign material contacts with blood, inducing of adsorbing proteins in blood, platelet adhesion and platelet activation cannot be avoided, then forming thrombus [28].

The results of platelet adhesion onto PES membranes are presented in Figure 6. It could be observed that the amount of adhering platelets decreased sharply with increasing the PVP-HLPU amounts in the modified membranes. When the PVP-HLPU content reached above $6 \mathrm{wt} . \%$ (M-6 and M8 ), there was almost no platelet adhesion on the modified membranes. Meanwhile, the adhered platelets on the surface of modified membranes showed rounded shape, and almost no pseudopodium and deformation were observed on the surface of the modified membrane. However, an aggregation of the adhered platelets on the original PES membrane were deformed with extended pseudopodia. It was due to the improved hydrophilicity and the relatively low protein adsorption on the modified membranes. It indicated that the hemocompatibility of the PVP-HLPU modified membranes was improved.

3.4.3. Clotting Times (APTT and TT). The activated partial thromboplastin time (APTT) and thrombin time (TT) are also used to characterize the anticoagulant properties of the membranes. We measured the APTTs and TTs for the membranes to investigate the anticoagulation activity of the membranes, and the results are presented in Figure 7.

Both the APTTs and TTs increased sharply with increasing the blended PVP-HLPU amounts. When the blended

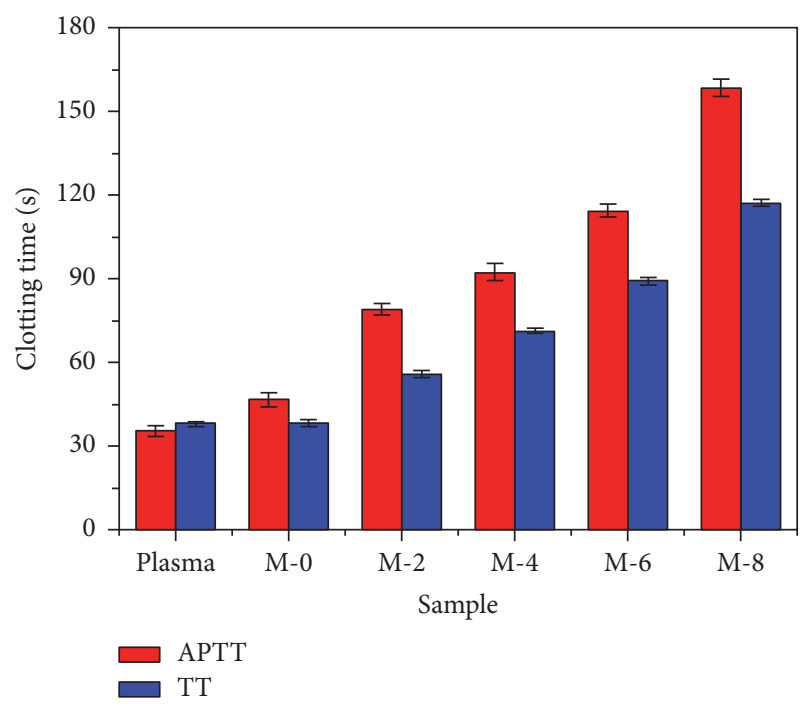

FIGURE 7: Clotting time results for all the membranes. Values are expressed as means $\pm \mathrm{SD}(n=3)$.

amounts of PVP-HLPU reached 8 wt.\%, both the APTT and TT of the membrane M- 8 were over three times longer than the APTT and TT of the membrane M- 0 . The increase of clotting time was much higher than those reported in other studies [29]. The excellent anticoagulant property was caused by the heparin-mimicking structure of HLPU, the enhanced hydrophilicity, and antifouling properties. Therefore, the clotting time tests indicated the anticoagulant properties of the PVP-HLPU modified membranes were improved.

3.4.4. Platelet Activation and Thrombin Generation. Contact activation of the coagulation system is crucial to induce blood coagulation. Platelet activation could result in platelet 

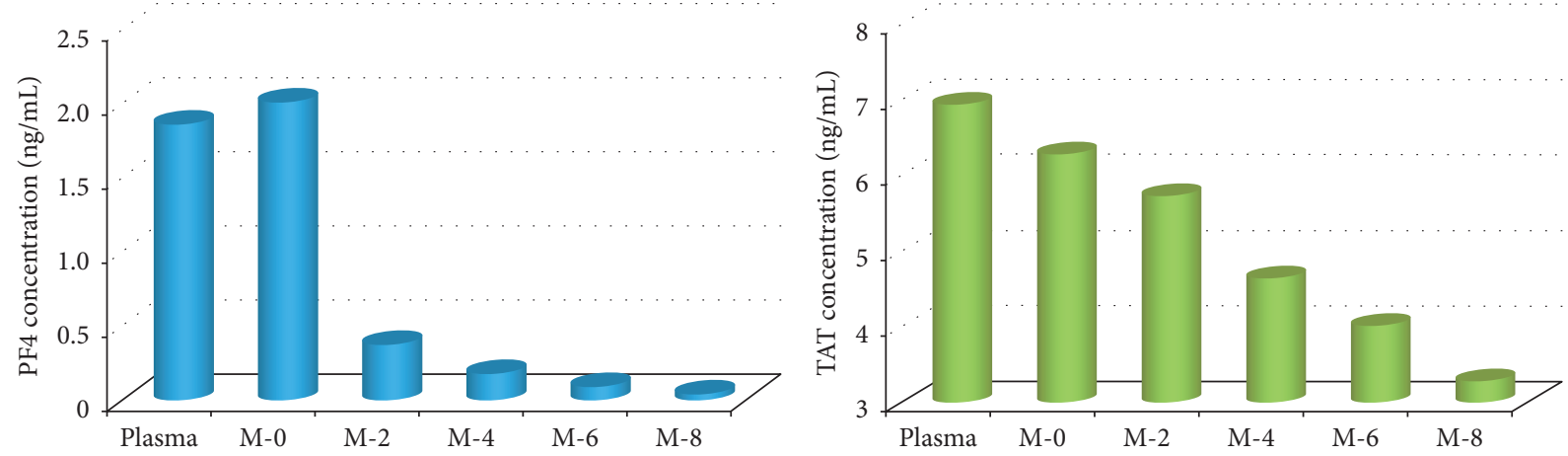

FIGURE 8: Platelet factor 4 (PF4) and thrombin-antithrombin III (TAT III) complex concentrations for the membranes.
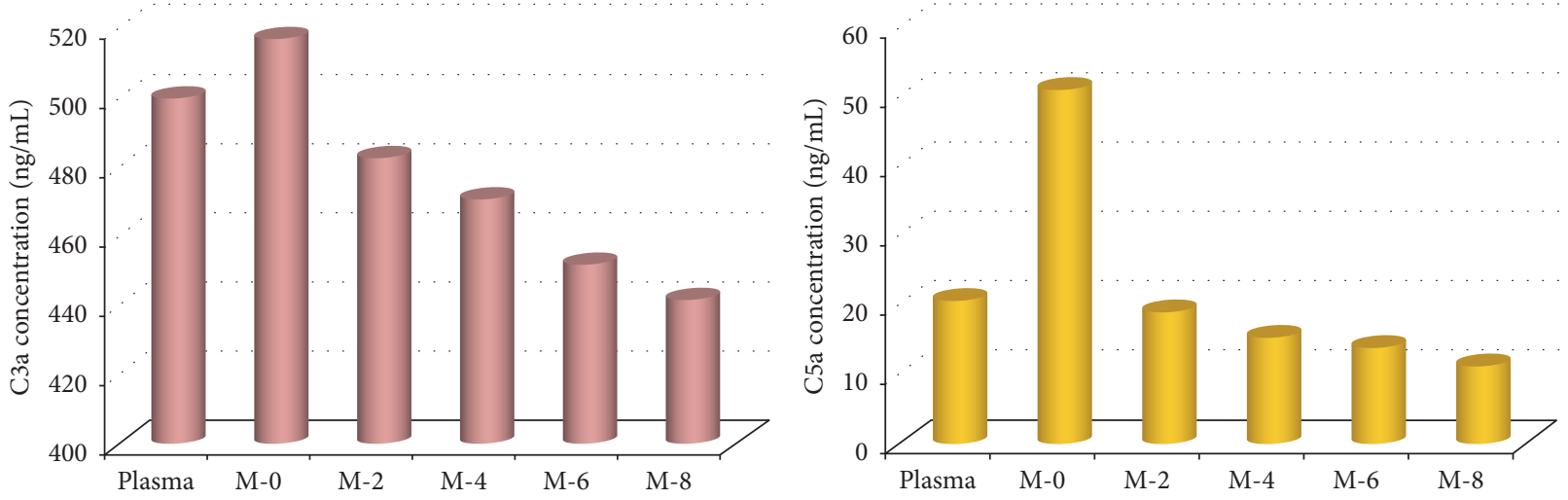

FIGURE 9: C3a and C5a concentrations for the membranes.

aggregation and activate the coagulation cascade system. Platelets interact with coagulation factors, while formed thrombin is a potent platelet-activating agonist [30]. The activated platelets could result in forming platelet factor 4 (PF4), while the formed thrombin could couple with antithrombin III to generate thrombin-antithrombin III (TAT) complexes [31]. Therefore, the concentrations of PF4 and TAT complexes could reflect the contact activation level, and the results are presented in Figure 8.

Both the PF4 and TAT complexes concentrations for the modified membranes decreased dramatically compared to the pristine PES membrane (M-0) or the plasma, which indicated that the modified membranes would not induce the activation of platelet and coagulation cascade. Thus, the modified membranes showed excellent blood compatibility after introducing the PVP-HLPU.

3.4.5. Complement Activation. Human complement system is also important in the body's defense mechanisms against infection and "nonself" elements [32]. After contacting the blood, the complement activation reflects the hemocompatibility of materials. After complement activation, followed by $\mathrm{C} 3 \mathrm{a}, \mathrm{C} 4 \mathrm{a}$, and $\mathrm{C5a}$ release, which are anaphylatoxins [33]. In current study, activation of C3a and C5a was tested to evaluate the complement activation of the modified membranes, and the results are presented in Figure 9.

Both the C3a and the C5a concentrations in plasma increased after the membrane M-0 contacted with the plasma. However, for the modified membranes, the C3a and the $\mathrm{C} 5 \mathrm{a}$ concentrations in plasma decreased to a certain extent. In addition, their concentrations decreased with increasing the amount of PVP-HLPU additive. The results indicated that the modified membranes showed suppressed complement activation after introducing the PVP-HLPU.

3.4.6. Comparison of End-Capped Hydrophilic Segments. In order to study the influence of the HLPUs with different endcapped hydrophilic segments on membrane performances, CA- and PEG-capped HLPUs were also synthesized and used for the modification of PES membranes. The molecular structures of the HLPUs are illustrated in Scheme 2.

In the study, 8 wt.\% of CA-, PEG-, and PVP-capped HLPUs were blended with 18 wt.\% of PES, and the membranes were fabricated by a phase-inversion method and termed as M-CA-HLPU, M-PEG-HLPU, and M-PVP-HLPU, respectively. Pure PES membrane was also prepared as the reference and named as M-PES. The properties of PES membranes are listed in Table 3. The hydrophilicity, water flux, and $F_{\mathrm{RR}}$ of modified PES membranes increased after blending functional HLPUs. The PES composite membranes with PEG-capped HLPU exhibited lowest protein adsorption compared with those with other functional HLPUs. PVP-capped HLPU could endow PES membranes (M-PVPHLPU) with the best blood compatibility, which was demonstrated by the highest APTT and TT and the lowest platelet activation, TAT generation, and complement activation. 


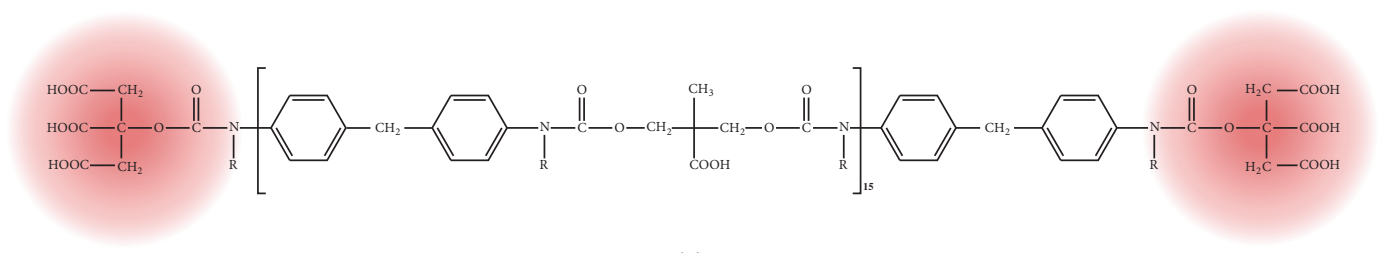

(a)

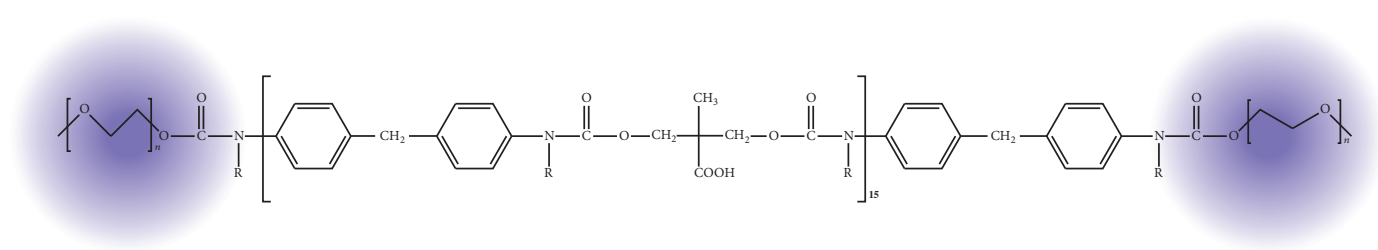

(b)

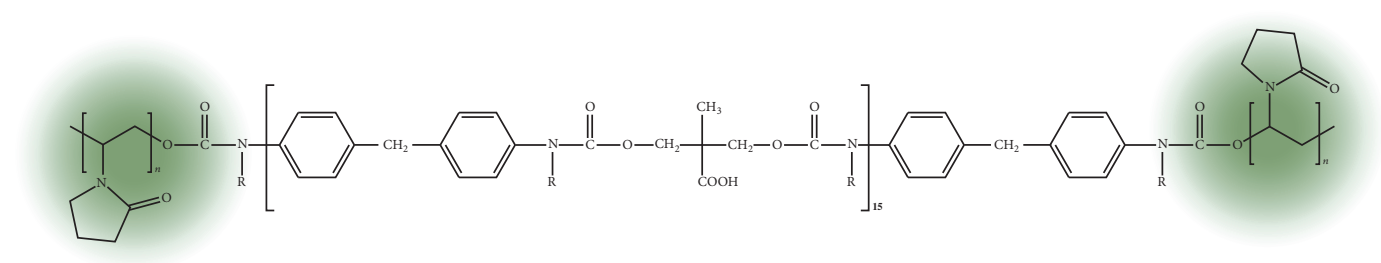

(c)

SCHEME 2: Molecular structures of HLPUs with different end-capped segments.

TABLE 3: Systematical comparison of different HLPUs modified membranes.

\begin{tabular}{|c|c|c|c|c|c|}
\hline Testing Name & Plasma & M-PES & M-CA-HLPU & M-PEG-HLPU & M-PVP-HLPU \\
\hline Contact angle $\left({ }^{\circ}\right)$ & I & 81.5 & 54.3 & 51.2 & 46.7 \\
\hline Water flux $\left(\mathrm{mL} / \mathrm{h} * \mathrm{~m}^{2} * \mathrm{mmHg}\right)$ & l & 30.6 & 165.2 & 175.5 & 155.1 \\
\hline$F_{\mathrm{RR}}(\%)$ & l & 40.8 & 95.3 & 95.3 & 92.0 \\
\hline BSA adsorption $\left(\mu \mathrm{g} / \mathrm{cm}^{2}\right)$ & l & 18.6 & 3.8 & 3.3 & 4.6 \\
\hline FBG adsorption $\left(\mu \mathrm{g} / \mathrm{cm}^{2}\right)$ & / & 12.1 & 2.7 & 1.6 & 2.5 \\
\hline $\operatorname{APTT}(s)$ & 35.5 & 46.8 & 136.2 & 100.2 & 158.7 \\
\hline $\mathrm{TT}(\mathrm{s})$ & 37.9 & 38.5 & 108.7 & 88.5 & 117.5 \\
\hline PF4 concentration (ng/mL) & 1.86 & 2.01 & 0.06 & 1.05 & 0.04 \\
\hline TAT concentration (ng/mL) & 6.95 & 6.29 & 5.05 & 4.86 & 3.29 \\
\hline C3a concentration $(\mathrm{ng} / \mathrm{mL})$ & 499.7 & 516.8 & 482.5 & 456.1 & 441.6 \\
\hline C5a concentration $(\mathrm{ng} / \mathrm{mL})$ & 20.6 & 51.1 & 16.9 & 13.9 & 11.2 \\
\hline
\end{tabular}

\section{Conclusion}

In this study, a heparin-like poly(vinyl pyrrolidone)-capped polyurethane (PVP-HLPU) was designed by reversible addition-fragmentation chain transfer polymerization combined with a sulfonation. The obtained PVP-HLPU could be directly blended with PES matrix to prepare membranes. The modified membranes showed excellent hemocompatibility and excellent protein antifouling properties compared with pristine PES membrane. In a conclusion, it is an effective system to evaluate the blood compatibility of the membranes by combination of APTT, TT, and platelet adhesion and so on. That is not to say that there is no drawback by use of these three methods. Other methods such as complement activation, hemolysis test could also be employed to characterize the blood compatibility of polymeric materials. Nonetheless, our results indicated that the PVP-HLPU modified membranes had great potential to be used in blood purification.

\section{Competing Interests}

The authors declare that they have no competing interests.

\section{Acknowledgments}

This work was financially sponsored by the National Natural Science Foundation of China (nos. 51225303 and 51433007).

\section{References}

[1] K. Sariibrahimoglu, W. Yang, S. C. G. Leeuwenburgh et al., "Development of porous polyurethane/strontium-substituted 
hydroxyapatite composites for bone regeneration," Journal of Biomedical Materials Research Part A, vol. 103, no. 6, pp. 19301939, 2015.

[2] A. T. Stevenson, L. M. Reese, T. K. Hill et al., "Fabrication and characterization of medical grade polyurethane composite catheters for near-infrared imaging," Biomaterials, vol. 54, pp. 168-176, 2015.

[3] Q. Chai, Y. Huang, and N. Ayres, "Shape memory biomaterials prepared from polyurethane/ureas containing sulfated glucose," Journal of Polymer Science, Part A: Polymer Chemistry, vol. 53, no. 19, pp. 2252-2257, 2015.

[4] Y.-J. Huang, K.-C. Hung, F.-Y. Hsieh, and S.-H. Hsu, "Carboxylfunctionalized polyurethane nanoparticles with immunosuppressive properties as a new type of anti-inflammatory platform," Nanoscale, vol. 7, no. 48, pp. 20352-20364, 2015.

[5] J. Cheng, X. Tang, J. Zhao, T. Shi, P. Zhao, and C. Lin, "Multifunctional cationic polyurethanes designed for non-viral cancer gene therapy," Acta Biomaterialia, vol. 30, pp. 155-167, 2016.

[6] C. Akduman, I. Özgüney, and E. P. A. Kumbasar, "Preparation and characterization of naproxen-loaded electrospun thermoplastic polyurethane nanofibers as a drug delivery system," Materials Science and Engineering: C, vol. 64, pp. 383-390, 2016.

[7] R. B. Trinca, G. A. Abraham, and M. I. Felisberti, "Electrospun nanofibrous scaffolds of segmented polyurethanes based on PEG, PLLA and PTMC blocks: physico-chemical properties and morphology," Materials Science \& Engineering C, vol. 56, pp. 511-517, 2015.

[8] F.-Y. Hsieh, H.-H. Lin, and S.-H. Hsu, "3D bioprinting of neural stem cell-laden thermoresponsive biodegradable polyurethane hydrogel and potential in central nervous system repair," Biomaterials, vol. 71, pp. 48-57, 2015.

[9] L. Rueda-Larraz, B. F. d'Arlas, A. Tercjak, A. Ribes, I. Mondragon, and A. Eceiza, "Synthesis and microstructure-mechanical property relationships of segmented polyurethanes based on a PCL-PTHF-PCL block copolymer as soft segment," European Polymer Journal, vol. 45, no. 7, pp. 2096-2109, 2009.

[10] R. Wang, T. Xiang, W.-F. Zhao, and C.-S. Zhao, "A facile approach toward multi-functional polyurethane/polyethersulfone composite membranes for versatile applications," Materials Science and Engineering: C, vol. 59, pp. 556-564, 2016.

[11] F. Zia, K. M. Zia, M. Zuber, S. Tabasum, and S. Rehman, "Heparin based polyurethanes: a state-of-the-art review," International Journal of Biological Macromolecules, vol. 84, pp. 101-111, 2016.

[12] M. Tan, Y. Feng, H. Wang et al., "Immobilized bioactive agents onto polyurethane surface with heparin and phosphorylcholine group," Macromolecular Research, vol. 21, no. 5, pp. 541-549, 2013.

[13] F. Kara, E. A. Aksoy, S. Calamak, N. Hasirci, and S. Aksoy, "Immobilization of heparin on chitosan-grafted polyurethane films to enhance anti-adhesive and antibacterial properties," Journal of Bioactive and Compatible Polymers: Biomedical Applications, vol. 31, no. 1, pp. 72-90, 2016.

[14] E. A. Aksoy, V. Hasirci, N. Hasirci, A. Motta, M. Fedel, and C. Migliaresi, "Plasma protein adsorption and platelet adhesion on heparin-immobilized polyurethane films," Journal of Bioactive and Compatible Polymers, vol. 23, no. 6, pp. 505-519, 2008.

[15] Y.-T. Hou, H. Ijima, T. Takei, and K. Kawakami, "Growth factor/ heparin-immobilized collagen gel system enhances viability of transplanted hepatocytes and induces angiogenesis," Journal of Bioscience and Bioengineering, vol. 112, no. 3, pp. 265-272, 2011.
[16] E. Brynda and M. Houska, "Multiple alternating molecular layers of albumin and heparin on solid surfaces," Journal of Colloid and Interface Science, vol. 183, no. 1, pp. 18-25, 1996.

[17] C. Zhao, J. Xue, F. Ran, and S. Sun, "Modification of polyethersulfone membranes-a review of methods," Progress in Materials Science, vol. 58, no. 1, pp. 76-150, 2013.

[18] C. Zhao, S. Nie, M. Tang, and S. Sun, "Polymeric pH-sensitive membranes-a review," Progress in Polymer Science, vol. 36, no. 11, pp. 1499-1520, 2011.

[19] L. Ma, B. Su, C. Cheng et al., "Toward highly blood compatible hemodialysis membranes via blending with heparin-mimicking polyurethane: study in vitro and in vivo," Journal of Membrane Science, vol. 470, pp. 90-101, 2014.

[20] W. Zhao, Q. Mou, X. Zhang, J. Shi, S. Sun, and C. Zhao, "Preparation and characterization of sulfonated polyethersulfone membranes by a facile approach," European Polymer Journal, vol. 49, no. 3, pp. 738-751, 2013.

[21] R. Chan and V. Chen, "Characterization of protein fouling on membranes: opportunities and challenges," Journal of Membrane Science, vol. 242, no. 1-2, pp. 169-188, 2004.

[22] Y.-L. Su, W. Cheng, C. Li, and Z. Jiang, "Preparation of antifouling ultrafiltration membranes with poly(ethylene glycol)-graftpolyacrylonitrile copolymers," Journal of Membrane Science, vol. 329, no. 1-2, pp. 246-252, 2009.

[23] A. L. Lim and R. Bai, "Membrane fouling and cleaning in microfiltration of activated sludge wastewater," Journal of Membrane Science, vol. 216, no. 1-2, pp. 279-290, 2003.

[24] A. Rahimpour, S. S. Madaeni, S. Zereshki, and Y. Mansourpanah, "Preparation and characterization of modified nanoporous PVDF membrane with high antifouling property using UV photo-grafting," Applied Surface Science, vol. 255, no. 16, pp. 7455-7461, 2009.

[25] J. Kost, "Protein at interfaces II. Fundamentals and applications," Journal of Controlled Release, vol. 45, no. 1, p. 113, 1997.

[26] M. Rabe, D. Verdes, and S. Seeger, "Understanding protein adsorption phenomena at solid surfaces," Advances in Colloid and Interface Science, vol. 162, no. 1-2, pp. 87-106, 2011.

[27] H. Chen, L. Yuan, W. Song, Z. Wu, and D. Li, "Biocompatible polymer materials: role of protein-surface interactions," Progress in Polymer Science, vol. 33, no. 11, pp. 1059-1087, 2008.

[28] B. Sivaraman and R. A. Latour, "Delineating the roles of the GPIIb/IIIa and GP-Ib-IX-V platelet receptors in mediating platelet adhesion to adsorbed fibrinogen and albumin," Biomaterials, vol. 32, no. 23, pp. 5365-5370, 2011.

[29] H. Wang, T. Yu, C. Zhao, and Q. Du, "Improvement of hydrophilicity and blood compatibility on polyethersulfone membrane by adding polyvinylpyrrolidone," Fibers and Polymers, vol. 10, no. 1, pp. 1-5, 2009.

[30] J. W. M. Heemskerk, E. M. Bevers, and T. Lindhout, "Platelet activation and blood coagulation," Thrombosis and Haemostasis, vol. 88, no. 2, pp. 186-193, 2002.

[31] C. H. Gemmell, "Assessment of material-induced procoagulant activity by a modified russell viper venom coagulation time test," Journal of Biomedical Materials Research, vol. 42, no. 4, pp. 611-616, 1998.

[32] M. B. Gorbet and M. V. Sefton, "Biomaterial-associated thrombosis: roles of coagulation factors, complement, platelets and leukocytes," Biomaterials, vol. 25, no. 26, pp. 5681-5703, 2004.

[33] A. Remes and D. F. Williams, "Immune response in biocompatibility," Biomaterials, vol. 13, no. 11, pp. 731-743, 1992. 

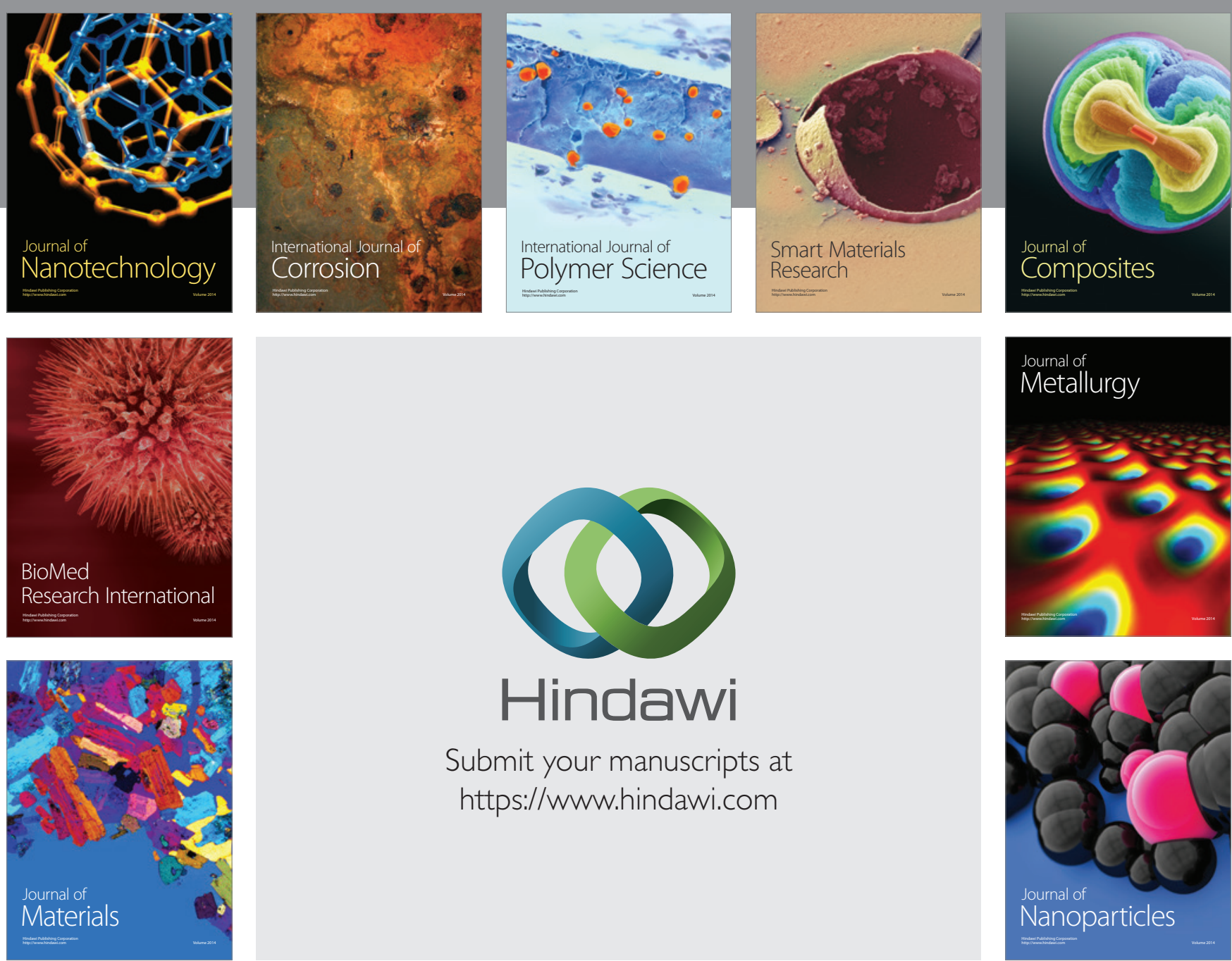

\section{Hindawi}

Submit your manuscripts at

https://www.hindawi.com

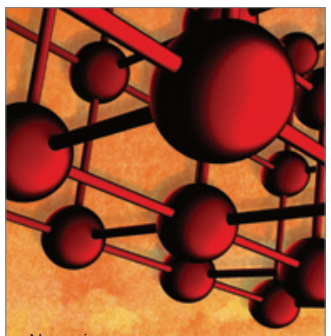

Materials Science and Engineering
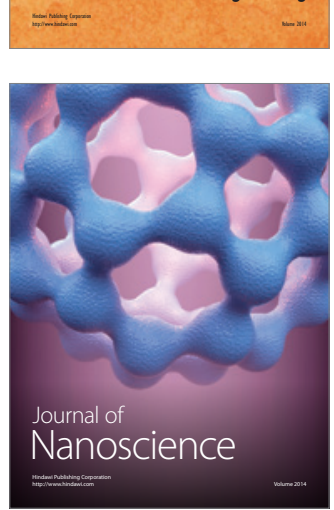
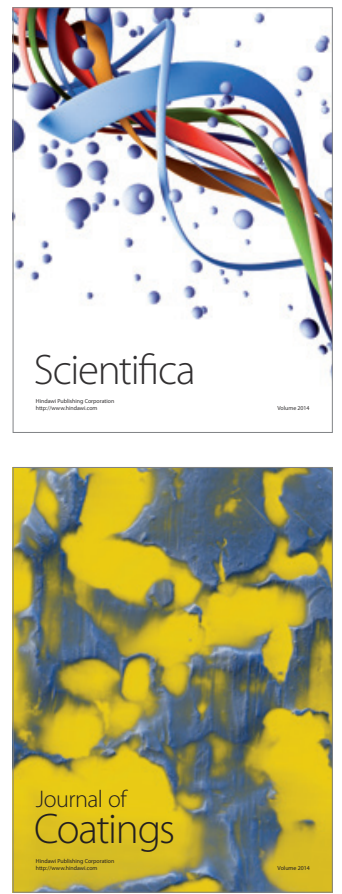
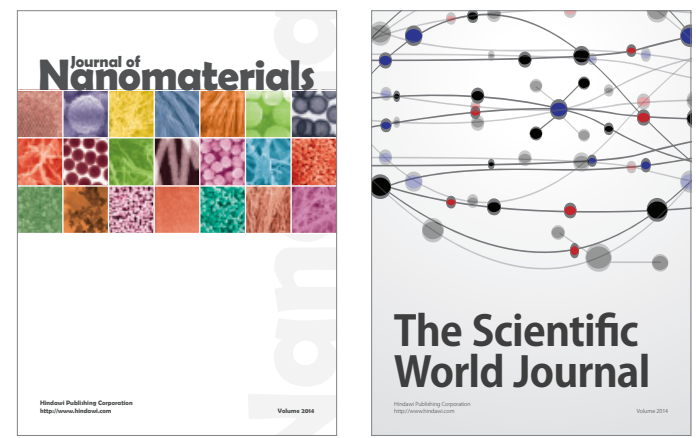

The Scientific World Journal
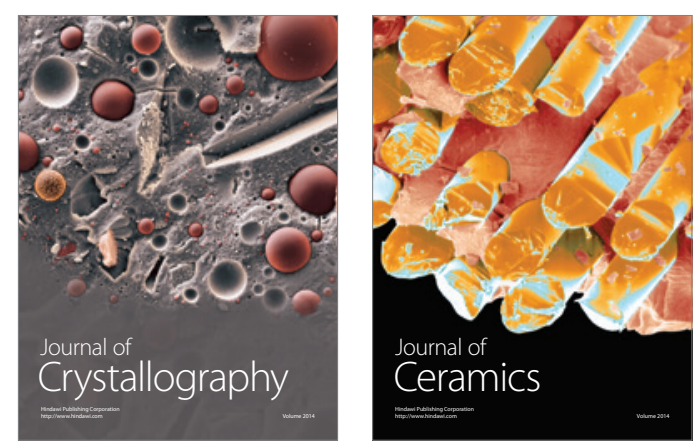
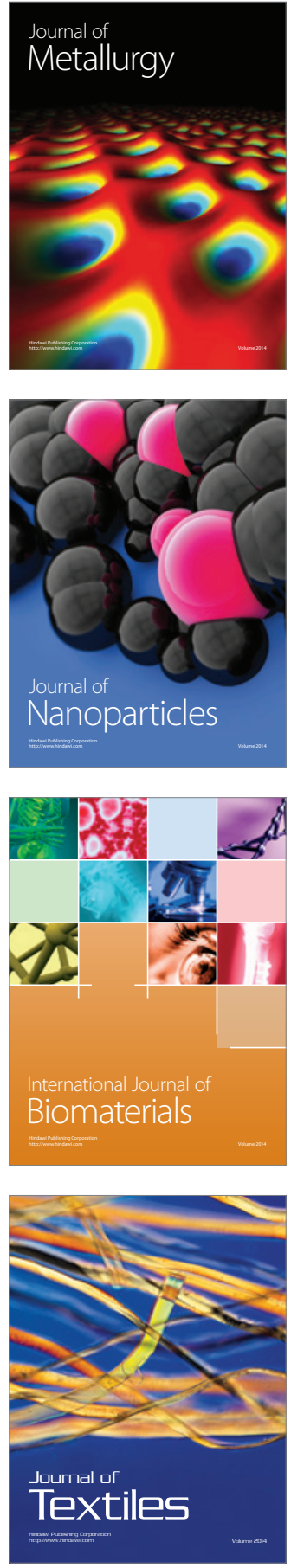